\title{
Dimensiones culturales del concepto de influenza humana en estudiantes y docentes de ciencias de la salud que favorecen o dificultan su prevención
}

\author{
Teresa Margarita Torres López, Osmar Juan Matsui Santana \\ y Carolina Aranda Beltrán
}

Se realizó un estudio de consenso cultural y de dimensiones conceptuales de la influenza A (HINI). La epidemia de influenza humana de 2009 ofrece la oportunidad de conocer el punto de vista de los actores implicados en el proceso de prevención y atención. Se aporta la visión de futuros profesionales de la salud y de docentes de ciencias de la salud. Los que favorecen la prevención del problema son los conceptos de influenza, síntomas, mecanismo de transmisión, cuidados sugeridos y su atención. Los factores que pueden obstaculizar la prevención son la falta de credibilidad en las autoridades y en la información transmitida por los medios de comunicación. Aquellas detectadas como posibles barreras para la prevención de la influenza deben combatirse con información científica suficiente y adecuada.

PALABRAS CLAVE: influenza humana, epidemia, consenso cultural, estudiantes del área de la salud, docentes

\section{Cultural Dimensions of Human Influenza Concept of Health Sciences Students and Docents that Allow or Difficult its Prevention}

The human influenza epidemic of 2009 gives us the opportunity to know the point of view of the actors involved on its care and prevention. This paper provides the view of health sciences students and docents. This is a consensus cultural and conceptual dimensions study of influenza A (HINI). The dimensions that help to prevent the problem were the concepts of influenza, symptoms, transmission and medical care. The possible barriers dimensions were the lack of credibility in the authorities and media messages. The possible barriers to prevent the influenza should be avoid with enough and adequate scientific information.

KEYWORDS: human influenza, epidemic, cultural consensus, students health occupations, faculty

\footnotetext{
Teresa Margarita Torres López: Centro Universitario de Ciencias de la Salud, Universidad de Guadalajara, Guadalajara, Jalisco, México tere.torres.cucs@gmail.com

Osmar Juan Matsui Santana: Centro Universitario de Ciencias de la Salud, Universidad de Guadalajara, Guadalajara, Jalisco, México omatsui@yahoo.com

Carolina Aranda Beltrán: Centro Universitario de Ciencias de la Salud, Universidad de Guadalajara, Guadalajara, Jalisco, México caranda2000@yahoo.com.mx
} 


\section{INTRODUCCIÓN}

$\mathrm{L}$ a epidemia de influenza humana de 2009 ofrece la oportunidad de conocer el punto de vista de los actores implicados en los procesos de prevención y atención de dicho evento. Este trabajo aporta la visión de futuros profesionales de la salud y de docentes de ciencias de la salud, $\mathrm{cu}^{-}$ yas concepciones culturales determinarán sus conductas de protección, prevención y atención ante la presencia de enfermedades emergentes, tanto en lo individual como en su práctica profesional. Existen pocos estudios relacionados con las concepciones de los profesionales de la salud en torno a la pandemia de influenza, a pesar de que se considera una población en mayor riesgo de contagio que la población en general. Hay dos trabajos sobre la aceptación de la vacunación contra dicha enfermedad en España, donde se reportó una baja cobertura (Sánchez-Payá et al., 2011) y sobre la baja intención de vacunarse (Apiñaniz et al., 2010). Respecto de la

1 percepción social y de las actitudes hacia la influenza A (H1N1) se realizó un estudio en la ciudad de México entre trabajadores del Instituto Nacional de Neurología y Neurocirugía, en el que se reportó que los trabajadores de la salud mostraron temor a contagiarse o que sus familiares contrajeran el padecimiento por esta vía y modificaron sus hábitos de higiene para cumplir con las medidas preventivas (Velásquez et al., 2009).

En un estudio previo a la pandemia de 2009 desarrollado en la costa este de Estados Unidos con trabajadores de la salud se encontró que su voluntad para reportarse en caso de una pandemia de influenza variaba según su puesto de trabajo. Los clínicos manifestaban mayor disponibilidad que otro tipo de trabajadores - técnicos y administrativosque se consideraban en un rol menos importante. $\mathrm{Al}$ parecer, la disposición positiva se relaciona con una preparación académica competente y con la disponibilidad del equipo de protección adecuado, así como con el soporte social y familiar pertinente durante la crisis. La no participación se asocia con la ambigüedad de la responsabilidad y de las tareas específicas durante la epidemia, lo mismo que con la percepción de carecer de las habilidades profesionales suficientes para enfrentar la contingencia (Balicer et al., 2006).

Una manera de acercarse a las percepciones y conocimientos sobre un objeto social es la teoría del consenso, cuya fundamentación se ubica en el campo de la antropología cognitiva, que estudia el proceso de adquisición de información sobre el mundo en una variedad de culturas: cómo la procesan, cómo toman decisiones y cómo actúan de una manera considerada apropiada por otros miembros de su cultura - transmisión cultural- (Bernard, 1996). La cultura es un conjunto de conocimientos, creencias y conductas compartidas que constituyen los elementos normativos de un grupo. La teoría del consenso es un conjunto de técnicas analíticas y modelos que pueden usarse para estimar los saberes culturales. Con base en esta teoría, el conocimiento individual, o competencia cultural individual, puede ser analizado desde el acuerdo entre los integrantes de un grupo determinado. Se parte de la idea de que las personas son culturalmente competentes en un área específica de un objeto social en la que son expertos no sólo por su conocimiento científico, sino también, y sobre todo, por los saberes culturalmente compartidos (Weller, 2007). En el tema del concepto de influenza se encuentran implicados conocimientos previos más los que se han incorporado después de la pandemia de influenza de 2009.

El modelo de la teoría del consenso parte de tres supuestos: 1) Cada informante debe proveer respuestas sobre el tema independientemente de los otros informantes, de tal forma que sus respuestas deben provenir de su punto de vista sin consultar la opinión de los otros - de ahí que la obtención de información se haga de manera individual-. 2) Debe tratarse un solo tema con el mismo nivel de dificultad para los participantes; la homogeneidad de cada palabra debe representar sólo un dominio de conocimiento y de competencia que deben ser consistentes. 3) Debe haber un alto nivel de acuerdo grupal 
en las repuestas obtenidas entre los informantes. Esta propuesta mide el acuerdo actual sobre un tema específico (Weller, 2007). El objetivo de este estudio fue describir las concepciones culturales sobre la influenza humana de estudiantes y docentes del Centro Universitario de Ciencias de la Salud (cucs) de la Universidad de Guadalajara (UdeG), con la finalidad de identificar dimensiones culturales que pudieran favorecer o no la prevención de la enfermedad.

\section{METODOLOGÍA}

Se realizó un estudio bajo el enfoque de la antropología cognitiva. La codificación de la información implica un tratamiento estadístico para obtener el análisis de consenso: se lleva a cabo un análisis cuantitativo de datos cualitativos - convertir palabras o imágenes en números- (Bernard, 1996). Participaron estudiantes y docentes del cucs, seleccionados por muestreo propositivo (Arcury y Quandt, 1998), conveniencia y facilidad de acceso. Para el ciclo de estudios 2010-2011, el cucs contó con 15276 estudiantes y 1772 miembros del personal académico. Desarrolla 89 programas académicos de niveles técnico, licenciatura - en cultura física y deportes, enfermería, médico cirujano y partero, nutrición, cirujano dentista y psicología-, especialidades - odontológicas y médicas - y posgrados (CuCs, 2011).

El tamaño de la muestra se basó en el supuesto del modelo de consenso para el estudio de patrones culturales: no debe ser grande porque la correlación promedio entre informantes tiende a ser alta -0.5 o más-. Los dos factores que determinan el número de informantes son la competencia cultural esperada - concordancia entre las respuestas individuales y el patrón estimado de respuestas correctas del grupoy el nivel de confianza aceptable para determinar la respuesta a la pregunta. Romney, Batchelder y Weller (1987) sugieren un tamaño mínimo de 17 informantes para clasificar $95 \%$ de las preguntas correctamente, bajo un promedio de competencia cultural esperada de 0.5 y un nivel de confianza de 0.95 . El trabajo tuvo dos recolecciones sucesivas de datos entre octubre y diciembre de 2009: una con la técnica de listados libres y otra con la técnica de sorteo de montones - pile sort- (Bernard, 2006). Participaron 917 estudiantes $-67.4 \%$ fueron mujeres y $32.6 \%$ hombres, con edades promedio de 21.4 y 20 años respectivamente - y 164 docentes $-50.8 \%$ eran hombres y $41.2 \%$ mujeres, con promedio de edad de 44.3 años en mujeres y 50.3 en hombres-.

En la primera fase del estudio se aplicó la técnica de listados libres (Bernard, 2006), que consiste en pedir a los informantes una lista por escrito de términos o frases relacionados con una esfera conceptual particular. Usamos las palabras relativas a influenza H1N1 y una definición de cada término. Esto se llevó a cabo por medio de una encuesta autoadministrada por computadora-Computer selfadministered questionnaire, CSAQ por sus siglas en inglés-(Díaz de Rada, 2000), a la que tuvieron acceso estudiantes y docentes, por medio de una invitación colectiva enviada por correo electrónico. Luego de obtener las listas de palabras, se tabularon los términos por frecuencia de mención. Se escogieron 20 palabras de cada lista para generar el instrumento de recolección de sorteo de montones aplicado en la segunda fase del estudio. Un segundo análisis fue la categorización de las definiciones dadas para cada palabra, lo cual se hizo con análisis de contenido temático (Minayo, 1995).

El sorteo de montones (Bernard, 2006) consiste en elaborar tarjetas para cada término y pedir a los informantes de ambos sexos que formen grupos de tarjetas que contengan las palabras más parecidas, sin importar el número de grupos generados. Después se les pide que etiqueten cada montón con una palabra que identifique el conjunto de las tarjetas seleccionadas. Los investigadores completaron esta fase de manera personalizada. Se aplicó análisis de consenso mediante la factorización de componentes principales -análisis dimensionales - por conglomerados jerárquicos y escalas multidimensionales. Con este último análisis se calculó el valor de estrés como 
medida de bondad de ajuste de la representación de los conglomerados. Esta medida se define como la suma de correspondencias entre pares de objetos dentro de la representación espacial. Sturrock y Rocha (2000) sugieren para una representación de 20 términos un valor de corte de estrés menor a 0.2. Los análisis se procesaron con el programa computarizado Anthropac v. 4.98 (Borgatti, 1996).

El proyecto de investigación que fundamentó el presente estudio fue evaluado y aprobado por el Comité de Ética e Investigación del Instituto de Investigación en Salud Ocupacional del Departamento de Salud Pública de la udeg — número de registro IISO/CI/03/10-. Antes de cada entrevista se explicaron los objetivos del estudio y se obtuvo el consentimiento informado verbal, además de que se garantizó a los informantes la confidencialidad y anonimato en la recolección y el análisis de la información. La participación de los entrevistados y entrevistadas se sujetó a su aceptación voluntaria.

\section{RESULTADOS}

\section{Estudiantes}

Los estudiantes de ciencias de la salud reportaron 4545 términos asociados con el concepto de influenza. Se agruparon en cinco categorías para la realización del análisis temático: concepto incluyó enfermedad, virus, cerdos; síntomas, elementos epidemiológicos, muerte, epidemia, contagio, etc.; prevención y cuidados, atención, hospital, salud, etc., y consecuencias sociales, muerte, miedo, crisis, etc. Las descripciones de estas categorías fueron:

Concepto: Es una enfermedad similar a la influenza A, se manifiesta como un padecimiento de las vías respiratorias, puede complicarse con neumonía y pone en severo peligro la salud y la vida del paciente si no se trata a tiempo. El agente etiológico es un virus nuevo, mutado, de la familia ortomixovirus, llamado virus de influenza A (H1N1). En un principio se manejó como fiebre porcina, ya que "la cepa que se presentó fue una variante de la influenza porcina".

Sintomas: Son los síntomas de resfriado común o de una gripa fuerte, agresiva, complicada, severa y aguda. El principal síntoma es la fiebre de más de $39^{\circ} \mathrm{C}$ más tos, inflamación de la garganta, escurrimiento nasal, estornudos, dolor de cabeza, malestar general y diarrea.

Elementos epidemiológicos: Es una enfermedad altamente infecto-contagiosa. Se transmite de persona a persona a través de gotas de saliva expelida por estornudos, tos o al hablar. Ataca a personas vulnerables, con bajas defensas. Se ha propagado con rapidez por la falta de higiene de la población. Causa la muerte si no se detecta o atiende a tiempo. Hay muchos casos de muertes y la mortalidad es más elevada que en otros tipos de influenza. Tiene magnitud de epidemia, afecta a gran parte de México y de otros países, por lo que se le cataloga como una epidemia mundial - universal-. El contagio se facilita por la capacidad de mutar del virus y por el cruce de fronteras debido a la movilidad humana. La Organización Mundial de la Salud (oms) declaró fase 4 o 5 de una contingencia sanitaria, lo que marcó un aislamiento social y anulación de actividades. Al parecer, la epidemia no está controlada.

Prevención y cuidados: La prevención ayuda a mantener la enfermedad controlada y evita su propagación. Son necesarias algunas medidas de protección e higiene. Se deben conocer sus bases científicas para manejarla mejor. Es conveniente tener cuidado de uno mismo y de los demás, sobre todo los prestadores de servicios de salud.

Cuidados sugeridos: Higiene, lavado de manos, uso de gel antibacterial, empleo de barreras de protección — cubrebocas, gafas y guantes-, uso de protección al salir a la calle, no saludar de mano ni de beso, desinfectar lugares de uso común, emplear cloro en la limpieza, no asistir a la escuela, aislar a enfermos y evitar acercarse a los infectados. Además, aplicar la vacuna y conservar el sistema inmunológico en buen estado.

Atención: Es necesario cuidar y mantener la salud de la población. En caso de enfermar, el tratamiento 
oportuno salva la vida del paciente. Medicamentos mencionados: antirretroviral o antiviral, Oseltamivir o Tamiflu.

Consecuencias sociales: Se percibe miedo al contagio de la influenza o al contagio de los seres queridos, también miedo a morir. Un alumno indicó: "Cuando alguien tose en el transporte público se siente miedo e incertidumbre".

Se duda de la existencia y de la gravedad de la epidemia o se considera una mentira por la falta de evidencias - cifras reales-, por no conocer a personas infectadas y por la información contradictoria recibida del gobierno y de los medios masivos de comunicación. Se plantea que existen problemas de salud más serios que la influenza humana. Se culpa al gobierno de usar la epidemia como una cortina de humo para desviar la atención y ocultar problemas más graves: aprobación de leyes, elecciones, crisis económica. Se piensa que la información emitida por los medios de comunicación es exagerada, lo que ha causado histeria colectiva y pánico entre la población, así como la discriminación de mexicanos en otros países. La epidemia de influenza se asoció con la crisis económica en dos sentidos. Como una forma de distracción de los problemas económicos nacionales y como causante de dicha crisis económica: no hay ventas, los negocios cierran y la actividad turística disminuye, todo lo cual deriva en desempleo. Los hombres expusieron dudas sobre la existencia y gravedad de la epidemia, mientras que las mujeres evidenciaron estar más involucradas en la prevención.

\section{Dimensiones de la influenza de los y las estudiantes}

Las dimensiones descritas en los árboles de conglomerados tuvieron valores de bondad de ajuste adecuados -estrés $=0.095$ en las alumnas y 0.079 en los alumnos- en el análisis de escala multidimensional no métrica. La agrupación de las alumnas se muestra en la figura 1. Pueden observarse tres dimensiones en el concepto de influenza: enfermedad, que incluye causas y proceso - consecuencias y síntomas-; consecuencias sociales, que abarca política y epidemiología, y prevención, que comprende cuidados y salud.

Las dimensiones registradas en los alumnos se muestran en la figura 2: enfermedad, que incluye proceso y síntomas; gobierno y niveles, que se refiere a amenaza y mal manejo de la información, y salud pública, que comprende prevención y cuidado.

\section{Docentes de ciencias de la salud}

Los docentes de ciencias de la salud reportaron 585 términos asociados con el concepto de influenza. $\mathrm{Pa}$ ra la realización del análisis temático se agruparon en las mismas cinco categorías utilizadas para los alumnos: concepto, sintomas, elementos epidemiológicos, prevención y cuidados, atención y consecuencias sociales. Las descripciones de las categorías fueron:

Concepto: Es una enfermedad respiratoria aguda, nueva y contagiosa, producida por un virus $-\mathrm{A}$ (H1N1) - o agente etiológico. Es una gripa fuerte. Por sus características infecto-contagiosas puede ser de magnitud epidémica o pandémica, pues se detecta en muchos países. El virus fue mutado de la gripe aviar y está asociado con la fiebre porcina.

Síntomas: Son los síntomas de una gripa, pero más fuerte -dolor de cabeza, fiebre, tos, estornudos, flujo nasal, cansancio, dolor de articulaciones, malestar-. Causa neumonía. Si no se cuida se agudizan los síntomas.

Elementos epidemiológicos: Afecta a poblaciones, países y continentes - diseminación mundial-. Es una alerta sanitaria que genera caos en el exterior del país. Se transmite por las gotas de saliva y por los objetos contaminados. Hay riesgo de morir si no se toman las medidas adecuadas. Un docente indicó: "Al parecer en nuestro medio las defunciones han ocurrido por ineficiencia de nuestro sistema de salud y las condiciones de salud de la población afectada”. 
Figura 1. Dimensiones del concepto de influenza de alumnas de ciencias de la salud, Guadalajara, México, 2009

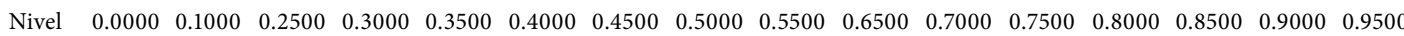

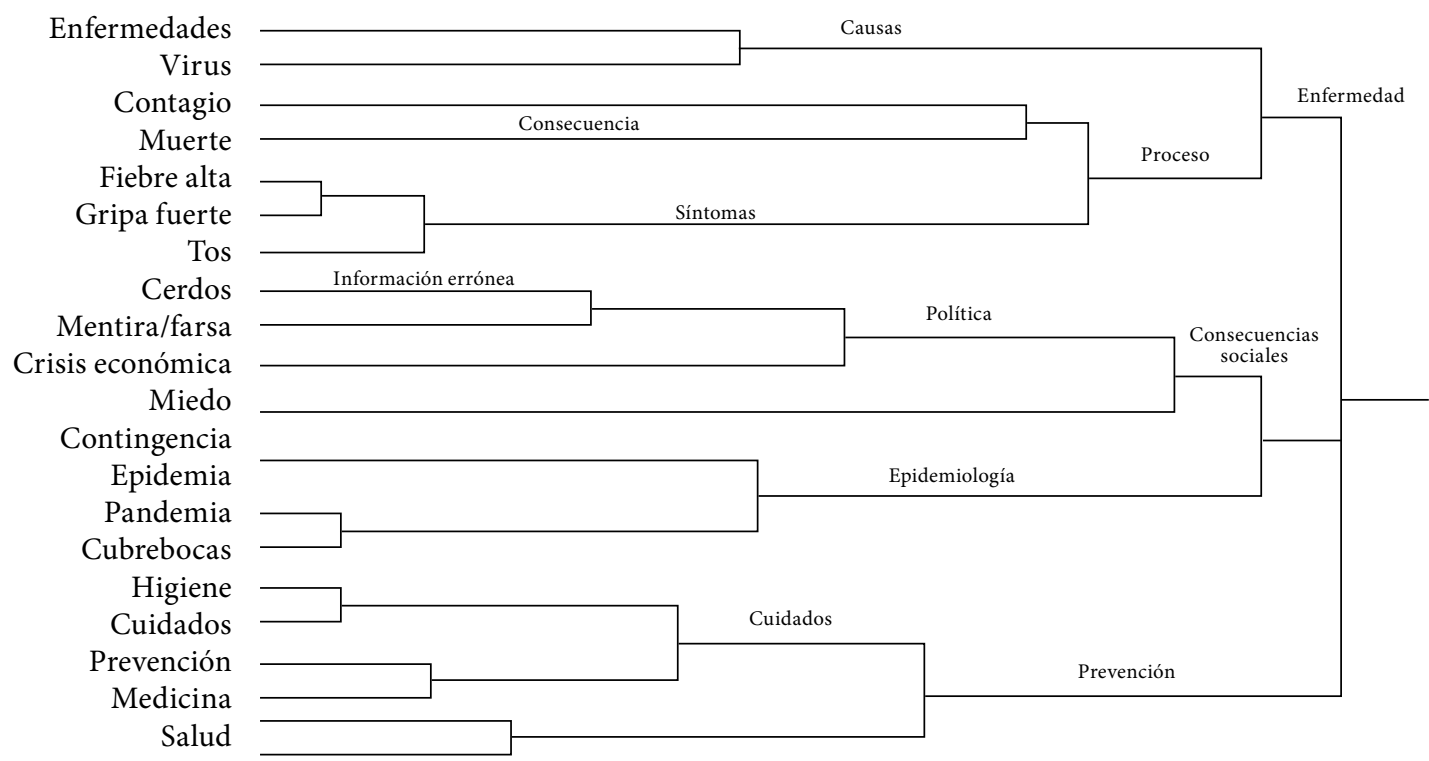

Prevención y cuidados: Con la prevención se detiene su diseminación. Se debe evitar el contagio, por ello es obligación personal cuidar de no contagiarse ni contagiar a otros. Cuidados sugeridos: higiene, cubrebocas - "para protegerse" - y tener un estilo de vida saludable. La vacuna es una posibilidad de defensa, pero no la hay. Alimentación sana, ventilación de los lugares, en áreas de concentración mantener distancia, cambio de ropa cuando se llegue a casa de la calle. Una mujer dijo: "Hay personas con gripe que nos infectan a todos, son las que deben quedarse en su casa o traer cubrebocas".

Atención: Acudir al médico para una atención médica apropiada, no usar remedios caseros, sino la medicina adecuada en tiempo y dosis. En caso de tener alguna duda y/o sintomatología no se debe automedicar. Medicamentos mencionados: Oseltamivir, Zanamivir, antibióticos para neumonía agregada.

Consecuencias sociales: Algunos de los participantes consideraron que se vive en riesgo, ya que todos estamos expuestos por tratarse de una enfermedad de peligro. La gente no toma en serio las medidas, son necesarias la participación y la responsabilidad de todos. Otros dudan de la existencia de la epidemia debido a la falta de evidencias sólidas. La información recibida es de corte autoritario, poco clara — “dicen mentiras”- y genera controversia. Está generando terror, miedo de contagio y muerte, pánico y comportamientos irracionales. Se afectan las relaciones interpersonales. Un hombre dijo al respecto: "Alguien estornuda y me retiro del lugar".

Hay efectos económicos por el cierre de las industrias y de las actividades laborales. Genera crisis en general y los más pobres sufren pérdidas de empleo. Ha provocado discriminación de mexicanos a nivel internacional. Hay desconfianza de las autoridades de salud y del gobierno en general - "hay negligencia”- Se presta para manejos políticos y ocultamiento de información. Los hombres externan pocas dudas sobre la existencia de la epidemia y hacen hincapié en la poca preparación del sistema de salud ante la magnitud del problema. Las mujeres se 
muestran más concentradas en la prevención, los cuidados de la enfermedad y la importancia de responsabilizar a toda la población en ello.

\section{Dimensiones de la influenza de los y las docentes}

Las dimensiones descritas en los árboles de conglomerados tuvieron valores de bondad de ajuste adecuados -estrés $=0.107$ en las docentes mujeres y 0.1 en los docentes- en el análisis de escala multidimensional no métrica. La agrupación que realizaron las mujeres se expone en la figura 3. Se observan tres dimensiones: enfermedad, que incluye epidemiología y sintomas; salud, que comprende prevención y responsabilidad del gobierno; y consecuencias, que abarca presión social —desconocimiento y rumoresy alarma.

Las dimensiones advertidas entre los docentes varones se detallan en la figura 4:1) enfermedad incluye síntomas, atención y control social,2) salud comprende prevención y cuidados; 3) percepción social abarca medios de información, temores y creencia popular.

\section{Consenso cultural sobre el concepto de influenza de estudiantes y docentes}

En el cuadro 1 se observa que el consenso cultural sobre el concepto de influenza fue elevado tanto entre los estudiantes como entre los docentes de ciencias de la salud. Sin embargo, el consenso fue mayor en estudiantes que en docentes y el nivel de consenso más bajo se registró entre los docentes hombres. Entre las alumnas alcanzó una razón de 11.1 y de 10.7 entre los alumnos. Entre las docentes mujeres fue de 9.6 y entre los docentes varones de 7.5. Se considera estadísticamente significativo que el primer factor abarque tres veces o más la varianza del segundo factor, lo cual sucedió en todos los casos.

Figura 2. Dimensiones del concepto de influenza de alumnos de ciencias de la salud,

Guadalajara, México, 2009

$\begin{array}{lllllllllllll}\text { Nivel } & 0.0000 & 0.1500 & 0.2500 & 0.3500 & 0.5500 & 0.6000 & 0.6500 & 0.7000 & 0.7500 & 0.8000 & 0.9000 & 1.0000\end{array}$

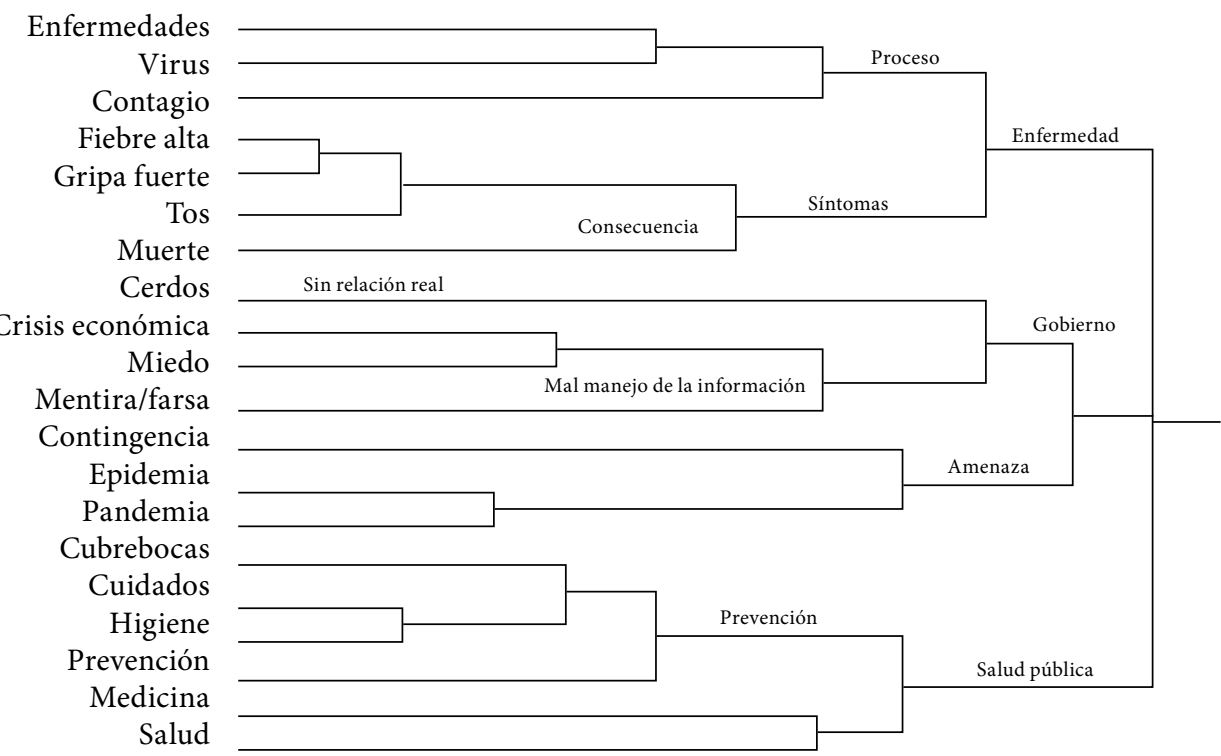


Figura 3. Dimensiones del concepto de influenza de mujeres docentes de ciencias de la salud, Guadalajara, México, 2009

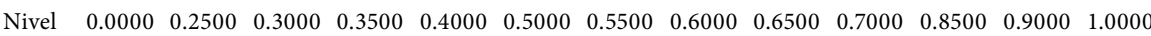

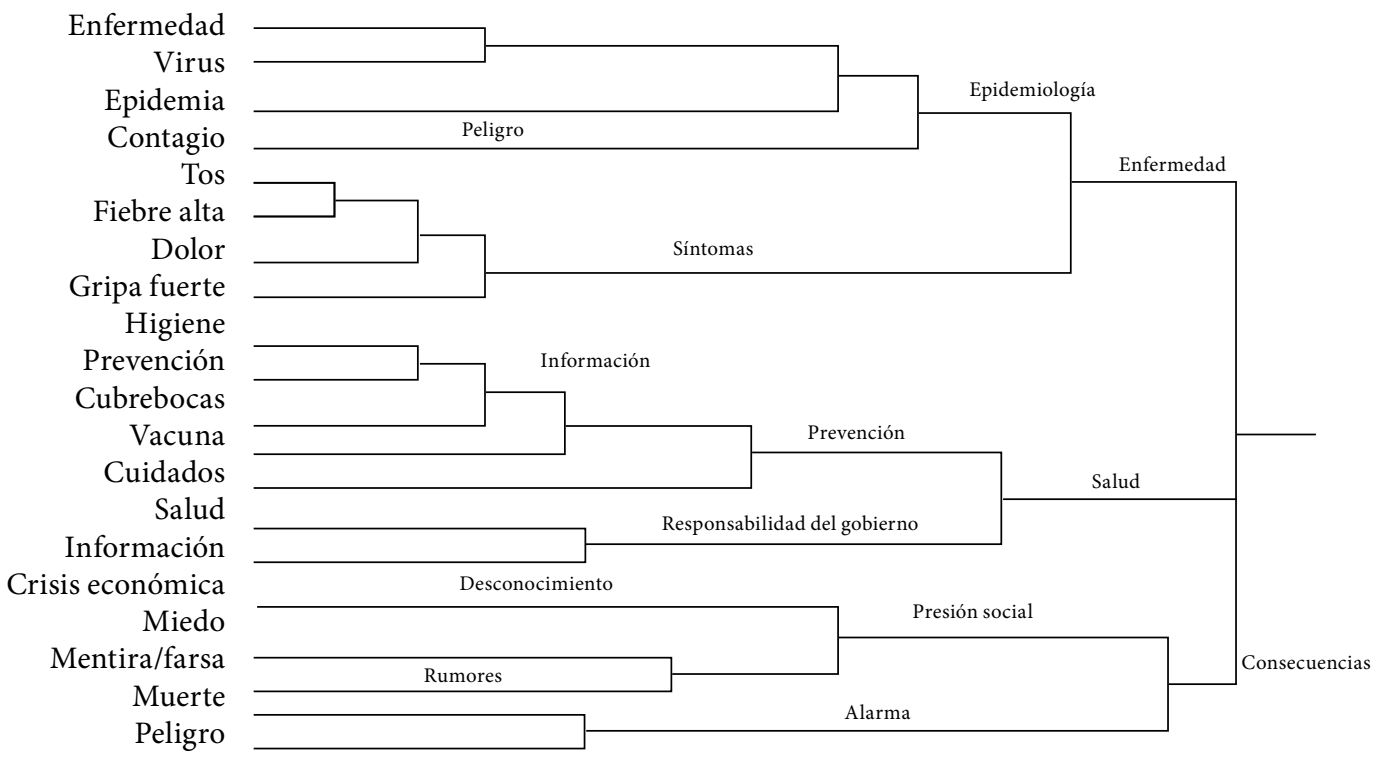

El porcentaje acumulado de la varianza para la razón entre los factores $\mathrm{F} 1$ y F2 ${ }^{1}$ fue mayor a $70 \%$. La media de la competencia cultural individual - media aritmética grupal del grado de acuerdo individual con las respuestas promedio- fue mayor a 0.50. Los valores confirman el consenso cultural (Weller, 2007). Los valores mayores a 0.3 en la medida de acuerdo grupal en las respuestas obtenidas por los informantes describen homogeneidad en la organización conceptual de las dimensiones y validan también el consenso. Esta cifra se obtiene elevando al cuadrado el promedio de la competencia cultural (Weller, 2007).

\section{DISCUSIÓN}

Se observaron dimensiones que favorecen la prevención y el manejo adecuado del problema, así como

\footnotetext{
${ }^{1}$ Estos factores corresponden a los dos primeros supuestos de la teoría del consenso citados en la introducción.
}

otras que representan posibles barreras para ello. Para los y las estudiantes las dimensiones que podrían favorecer la prevención son las del concepto de influenza, los sintomas, el mecanismo de transmisión, los cuidados y la atención. Se destacó la prevención como un elemento importante para el control de la enfermedad. Los altos niveles de consenso muestran la concordancia grupal en torno al concepto de influenza, que se conforma con información que pudo provenir de diversas fuentes, entre otras su formación académica. La identificación de la influenza como un riesgo suele ser mayor en personas que tienen contacto con profesionales de la salud (Yap et al., 2010).

Entre las dimensiones que podrían ser barreras para la implementación de prácticas preventivas están aquellas que referían que la epidemia estaba fuera de control - percepción social documentada por otros autores- (Cisneros, 2010), la duda de la existencia de la epidemia y la difusión de información exagerada y errónea en torno a la influenza - el señalamiento sobre el manejo de información alarmista de los medios 
de comunicación, sobre todo en relación con las cifras de contagiados y de muertes-. Esto se observó a partir de un análisis de las publicaciones de la prensa en el estado de Jalisco (Valdez, Díaz y Huerta, 2010), cuyos resultados son similares a los reportados por la prensa española en torno al tema (Camacho, 2009).

La falta de credibilidad en las autoridades respecto del manejo de medidas preventivas extremas, como el aislamiento social, fue otra dimensión cultural que se cree que podría obstaculizar la prevención del padecimiento. Esta falta de credibilidad fue referida en la India (Kamate et al., 2010), mientras que en otros países, como Reino Unido y Australia, la población manifestó tener confianza en sus representantes (Rubin et al., 2009; Seale et al., 2009).

En lo que corresponde a las diferencias entre los y las estudiantes, se observó que los hombres albergaban más dudas sobre la gravedad de la epidemia y menos conocimiento de medidas específicas de prevención, lo que implica menor percepción del riesgo.
Algo similar se encontró entre la población de Australia y Hong Kong (Seale et al., 2009; Cowling et al., 2010), lo que deriva en una pobre implementación de medidas preventivas de protección e higiene (Rubin et al., 2009). Las mujeres estudiantes refirieron mayor variedad de términos asociados con el concepto de influenza y señalaron más medidas preventivas. Un mayor interés sobre la prevención de la enfermedad fue encontrado entre mujeres con alta escolaridad de China (Lin et al., 2011) y entre las jóvenes de 18 a 24 años en Reino Unido (Rubin et al., 2009).

En el caso de los docentes las dimensiones positivas para la prevención de la influenza fueron el concepto de la enfermedad, los síntomas, el mecanismo de transmisión, riesgo de muerte, los cuidados y la atención que se debe recibir. Como se mencionó, la identificación de la influenza como un riesgo suele ser mayor entre profesionales de la salud (Apiñaniz et al., 2010) e implicar mejores prácticas preventivas, como hábitos y medidas de higiene

Figura 4. Dimensiones del concepto de influenza de hombres docentes de ciencias de la salud,

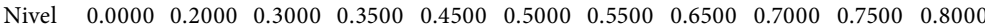

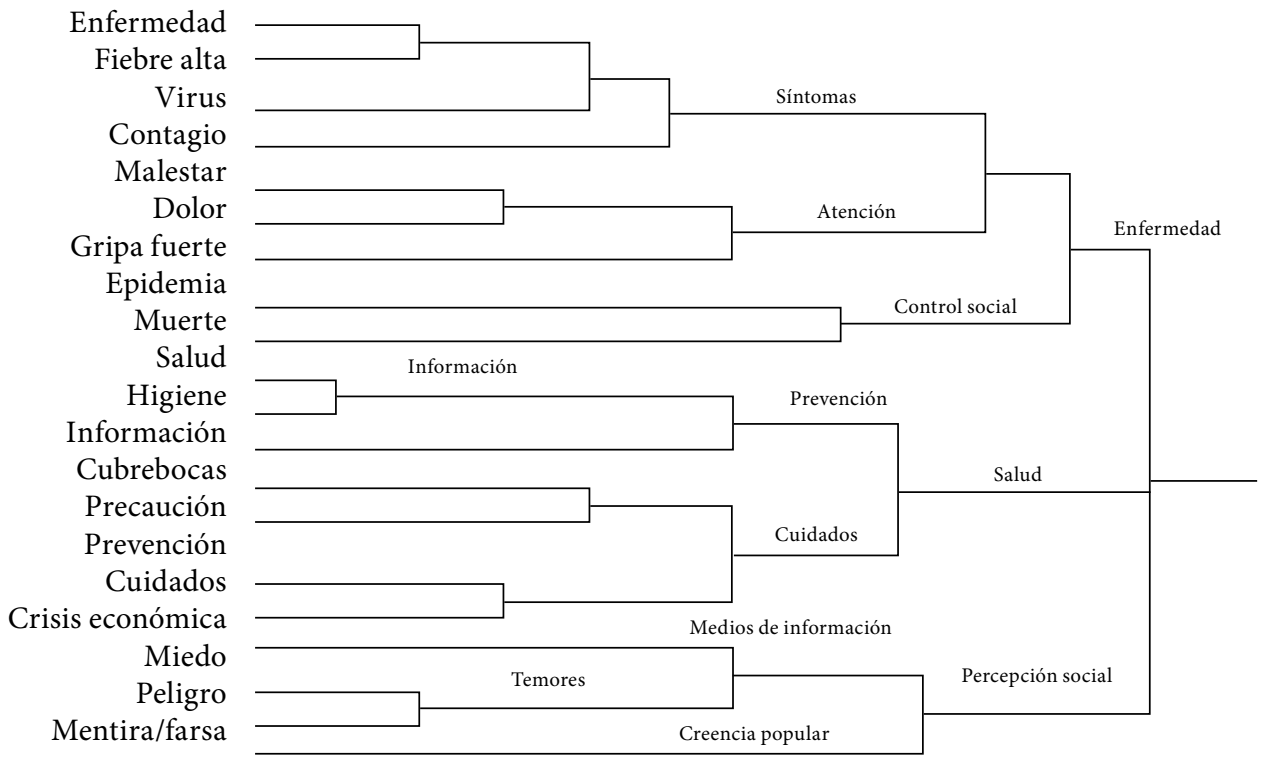


(Velásquez et al., 2009). En Australia se detectó que cuando la población tiene un conocimiento básico de la enfermedad hay mayor cumplimiento de las medidas preventivas (Eastwood et al., 2009). La identificación clara del rol como profesionales de la salud se relaciona con un vínculo mayor con el problema, como se puso de manifiesto entre el personal médico de la costa este de Estados Unidos (Balicer et al., 2006). Los docentes insistieron en que su papel era de formadores de futuros profesionales y de educadores de la población en general.

Los y las docentes señalaron una de las dimensiones que puede ser una barrera para una adecuada prevención de la influenza: la implementación de las medidas de aislamiento social implicó la afectación de las relaciones interpersonales, mantener la distancia de las personas en lugares públicos, la reducción del contacto físico y el uso de barreras protectoras, como el cubrebocas. Al igual que lo reportado en población de Hong Kong, al inicio de la epidemia, cuando la población tenía altos niveles se relajaron (Lin et al., 2011; Lau et al., 2009). En el caso de la población de la India, se redujo su asistencia a espacios públicos durante la contingencia (Kamate et al., 2010), mientras que en Reino Unido no se registró cambio alguno en este sentido (Rubin et al., 2009).

Otra dimensión que podría ser una posible barrera para la prevención adecuada de la influenza es la información insuficiente en torno a la epidemia, lo que genera miedo y alarma entre la población. Estados emocionales como la incertidumbre y el miedo podrían ser un arma de dos filos en la prevención de la influenza. Si bien cierto nivel de ansiedad puede generar cambios positivos en las conductas de prevención, como sucedió en Malasia (Goodwin et al., 2009), un nivel elevado puede conducir a la paralización de la población ante una amenaza considerada inevitable (Barrera, 2010). Los docentes varones muestran pocas dudas sobre la existencia de la epidemia, aunque destacan la desconfianza en el papel ejercido por las autoridades, en particular sobre la eficiencia de los servicios de salud ante un problema de tal magnitud. Una percepción similar Cuando esta sensación disminuyó dichas medidas

Cuadro 1. Niveles de consenso cultural sobre el concepto de influenza entre estudiantes y docentes de ciencias de la salud, México, 2009

\begin{tabular}{|c|c|c|c|c|c|c|c|c|}
\hline Participantes & $\begin{array}{l}\text { Razón del } \\
\text { Factor } 1 \\
\quad(F 1)\end{array}$ & $\begin{array}{c}\text { Varianza } \\
\text { acumulada } \\
\%\end{array}$ & $\begin{array}{l}\text { Razón del } \\
\text { Factor } 2 \\
\text { (F2) }\end{array}$ & $\begin{array}{c}\text { Varianza } \\
\text { acumulada } \\
\%\end{array}$ & $\begin{array}{l}\text { Razón } \\
\text { F1: F2 }{ }^{1}\end{array}$ & \multicolumn{2}{|c|}{$\begin{array}{c}\text { Competencia } \\
\text { cultural }^{2} \\
\text { Media } D E^{3}\end{array}$} & $\begin{array}{l}\text { Acuerdo } \\
\text { grupal }^{4}\end{array}$ \\
\hline $\begin{array}{l}\text { Alumnas cucs } \\
(\mathrm{n}=20)\end{array}$ & 11.0 & 88.4 & 0.7 & 94.5 & 14.6 & 0.73 & 0.11 & 0.53 \\
\hline $\begin{array}{l}\text { Alumnos cucs } \\
(\mathrm{n}=20)\end{array}$ & 10.7 & 88.2 & 0.8 & 94.6 & 13.8 & 0.72 & 0.10 & 0.52 \\
\hline $\begin{array}{l}\text { Docentes mujeres } \\
(\mathrm{n}=20)\end{array}$ & 9.6 & 84.5 & 1.1 & 94.3 & 8.6 & 0.68 & 0.12 & 0.46 \\
\hline $\begin{array}{l}\text { Docentes hombres } \\
(n=20)\end{array}$ & 7.5 & 80.3 & 1.1 & 92.5 & 6.5 & 0.56 & 0.24 & 0.31 \\
\hline
\end{tabular}

${ }^{1}$ Cociente de F1 en relación con F2. La regla es que F1 sea por lo menos tres veces mayor que F2 para hacer válido el consenso (Weller, 2007).

${ }^{2}$ Media aritmética grupal del grado de acuerdo individual con las respuestas promedio. Es la concordancia entre las respuestas individuales y el patrón estimado de respuestas correctas del grupo (Weller, 2007).

${ }^{3}$ Desviación estándar.

${ }^{4}$ Acuerdo grupal en las respuestas obtenidas por los informantes. Se obtiene elevando al cuadrado el promedio de la competencia cultural (Weller, 2007). 
predomina entre los estudiantes al destacar la visión de que la epidemia estaba fuera de control (Cisneros, 2010). Las mujeres docentes estaban más centradas en la prevención y los cuidados de la enfermedad, al igual que las estudiantes. Se encontraron divergencias de género equiparables entre la población universitaria de Corea (Park et al., 2010) y en el caso de mujeres de la India (Kamate et al., 2010). A ello se suma el señalamiento de las docentes sobre la importancia de involucrar a toda la población en estas estrategias. Visualizan el problema como un fenómeno global y centran la responsabilidad de su prevención no sólo en individuos o instituciones.

En conclusión, deben combatirse las dimensiones encontradas como posibles barreras para la prevención de la influenza con información científica suficiente y adecuada. El papel de los medios de comunicación en la prevención de la enfermedad es fundamental. Es indispensable que cuenten con preparación adecuada en materia de salud, como tomar en cuenta las variantes culturales de la población hacia la que dirigen sus mensajes (Vaughan y Tinker, 2009). La información científica considerada positiva y suficiente por la población reduce la incertidumbre y evita el pánico (Lin et al., 2011), facilita la percepción adecuada del riesgo y beneficia el desarrollo de prácticas preventivas (Rubin et al., 2009).

Este trabajo revela las limitaciones propias de los estudios del enfoque cognitivo en que se enfatizan los elementos cognoscitivos sin contemplar los niveles de análisis grupal, las prácticas y los procesos participativos. Este aspecto podría subsanarse al confrontar los resultados encontrados con el desarrollo de acciones específicas de los profesionales de la salud en materia de prevención de la influenza en su actividad profesional. Otra limitación es el tipo de muestreo utilizado: los resultados se restringen a las poblaciones estudiadas. Una de las aportaciones teórico-metodológicas de estudios con enfoque de la antropología cognitiva, como el presente, es mostrar una forma de acercamiento científico a las concepciones culturales de la población, con estrategias de recolección de información sencilla y más rápida que las entrevistas tradicionales. Así se accede de forma natural al punto de vista de los directamente implicados, por lo que el proceso metodológico es transferible a cualquier tipo de población local, nacional e internacional.

\section{Bibliografía}

Apiñaniz, Antxon et al., 2010, "Estudio transversal basado en la población sobre la aceptabilidad de la vacuna y la percepción de la gravedad de la gripe A (H1N1): opinión de la población general y de los profesionales sanitarios", en Gaceta Sanitaria, vol. 24, núm. 4, pp. 314-320.

Arcury, Thomas y Sara Quandt, 1998, "Qualitative Methods in Arthritis Research: Sampling and Data Analysis", en Arthritis Care Research, vol. 11, núm. 1, pp. 66-74.

Balicer, Ran et al., 2006, "Local Public Health Workers' Perceptions Toward Responding to an Influenza Pandemic", en BMC Public Health, vol. 6, núm. 99.

Barrera, Juan Antonio, 2010, "El miedo colectivo: el paso de la experiencia individual a la experiencia colectiva", en El Cotidiano, vol. 25, núm. 159, pp. 5-10, en línea: $<$ http://redalyc.uaemex.mx/src/inicio/ArtPdfRed. jsp?iCve $=32512747002>$.

Bernard H., Rusell, 1996, "Qualitative Data, Quantitative Analysis", en Cultural Anthropology Methods Journal, vol. 8, núm. 1, pp. 9-11.

, 2006, "Structured Interviewing II: Cultural Domain Analysis", en Rusell Bernard, Research Methods in Cultural Anthropology, Sage Publications, Londres, Nueva Delhi, pp. 299-317.

Borgatti, Stephen, 1996, Anthropac, Analytic Technologies, Lexington.

Camacho, Idoia, 2009, "La 'gripe A' en la prensa española", en Revista Latina de Comunicación Social, núm. 64, pp. 827-843, en línea: <http://www.revistalatinacs.org/09/ art/865_Bilbao/66_92_Idoia_Camacho.html $>$.

Centro Universitario de Ciencias de la Salud (cucs), 2011, Numeralia institucional, Centro Universitario de Ciencias de la Salud, Universidad de Guadalajara, Guadalajara, en línea: <http://www.cucs.udg.mx/categoria.php?id=476>.

Cisneros, José Luis, 2010, “25 días de miedo", en El Cotidiano, vol. 25, núm. 159, pp. 37-43, en línea: <http://redalyc. uaemex.mx/src/inicio/ArtPdfRed.jsp?iCve= 32512747006>. 
Cowling, Benjamin et al., 2010, "Community Psychological and Behavioral Responses through the First Wave of the 2009 Influenza A (H1N1) Pandemic in Hong Kong”, en The Journal of Infectious Diseases, vol. 202, núm. 6, pp. 867-876.

Díaz de Rada, Vidal, 2000, "Utilización de nuevas tecnologías para el proceso de 'recogida de datos' en la investigación social mediante encuesta”, en Revista Española de Investigaciones Sociológicas, núm. 91, pp. 137-166.

Eastwood, Keith et al., 2009, "Knowledge about Pandemic Influenza and Compliance with Containment Measures among Australians", en Bulletin of the World Health Organization, núm. 87, pp. 588-594, en línea: <http:// www.who.int/bulletin/volumes/87/8/08-060772.pdf $>$.

Goodwin, Robin et al., 2009, "Initial Psychological Responses to Influenza A (H1N1) ('Swineflu')", en BMC Infectious Diseases, vol. 9, núm. 166, en línea: <http://www. biomedcentral.com/content/pdf/1471-2458-6-99.pdf >.

Kamate, Shivlingesh et al., 2010, "Public Knowledge, Attitude and Behavioural Changes in an Indian Population during the Influenza A (H1N1) Outbreak", en Journal of Infection in Developing Countries, vol. 4, núm. 1, pp. 7-14.

Lau, Joseph et al., 2009, "Widespread Public Misconception in the Early Phase of the H1N1 Influenza Epidemic”, en Journal of Infection, núm. 59, pp. 122-127.

Lin, Yilan et al., 2011, "Knowledge, Attitudes and Practices KAP Related to the Pandemic (H1N1) 2009 among Chinese General Population: a Telephone Survey", en BMC Infectious Diseases, vol. 11, núm. 128, en línea: $<$ http://www.biomedcentral.com/content/pdf/14712334-11-128.pdf $>$.

Minayo, Cecília, 1995, El desafío del conocimiento. Investigación cualitativa en salud, Lugar Editorial, Buenos Aires, pp. 167-177.

Park, Jae-Hyun et al., 2010, "Perceptions and Behaviors Related to Hand Hygiene for the Prevention of H1N1 Influenza Transmission among Korean University Students during the Peak Pandemic Period", en BMC Infectious Diseases, vol. 10, núm. 222, en línea: <http://www. biomedcentral.com/1471-2334/10/222>.

Romney, A. Kimball, William H. Batchelder y Susan C. Weller, 1987, "Recent Applications of Cultural Consensus
Theory", en American Behavioral Scientist, vol. 31, núm. 2, pp. 163-77.

Rubin G., James et al., 2009, "Public Perceptions, Anxiety and Behaviour Change in Relation to the Swine Flu Outbreak: Cross Sectional Telephone Survey", en British Medical Journal, vol. 339, núm. b2651, en línea: <http:// www.bmj.com/content/339/bmj.b2651.full.pdf>.

Sánchez-Payá, José et al., 2011, "Determinantes de la vacunación antigripal en personal sanitario, temporada 2009-2010", en Gaceta Sanitaria, vol. 25, núm. 1, pp. 29-34.

Seale, Holly et al., 2009, “The Community's Attitude Towards Swine Flu and Pandemic Influenza”, en Medical Journal of Australia, núm. 191, pp. 267-269, en línea: <http://www.mja.com.au/public/issues/191_05_ 070909/sea10770_fm.html>.

Sturrock, Kenneth y Jorge Rocha, 2000, "A Multidimensional Scaling Stress Evaluation Table", en Field Methods, vol. 12, núm. 1, pp. 49-60.

Valdez, Andrés, Sergio Díaz y Delia Huerta, 2010, “Influenza y opinión pública en Jalisco: un estudio sobre las percepciones ciudadanas en Guadalajara, México", en Pangea, Revista de la Red Académica Iberoamericana de Comunicación, vol. 1, núm. 5, pp. 98-107, en línea: <http://dialnet.unirioja.es/servlet/articulo?codigo $=3405409>$.

Vaughan, Elaine y Timothy Tinker, 2009, "Effective Health Risk Communication about Pandemic Influenza for Vulnerable Populations", en American Journal of Public Health, núm. 99, pp. S324-S332.

Velásquez, Leora et al., 2009, "Percepción social y actitudes de los trabajadores del Instituto Nacional de Neurología y Neurocirugía ante la alerta epidemiológica de la influenza humana A (H1N1) en México durante 2009”, en Medicina Interna de México, vol. 25, núm. 6, pp. 450-453.

Weller, Susan, 2007, "Questions Cultural Consensus Theory: Applications and Frequently Asked", en Field Methods, núm. 19, pp. 339-368.

Yap, Jonathan et al., 2010, "Knowledge, Attitudes and Practices Towards Pandemic Influenza among Cases, Close Contacts and Healthcare Workers in Tropical Singapore: a Cross-Sectional Survey", en BMC Infectious Diseases, vol. 10, núm. 442. 